\title{
Mudanças recentes na Política Nacional de Atenção Básica: uma análise crítica
}

\author{
Recent changes in the National Primary Care Policy: a critical appraisal \\ Cambios recientes en la Política Nacional de Atención Primaria: una \\ evaluación crítica
}

Leonardo Ferreira Fontenelle ${ }^{1 \star}$

Palavras-chave Atenção Primária à Saúde Medicina de Família e Comunidade

Política de Saúde Qualidade, Acesso e Avaliação da Assistência à Saúde Distribuição de Médicos

Keywords: Primary Health Care

Family Practice

Health Policy

Health Care Quality

Access and Evaluation Distribution of Physicians

Palabras clave: Atención Primaria de Salud Medicina Familiar y Comunitaria

Política de Salud

Calidad, Acceso y Evaluación de la Atención de Salud Distribución de Médicos

\section{Resumo}

A nova Política Nacional de Atenção Básica (PNAB) mantém a essência do documento anterior, ao mesmo tempo que incorpora as inovações dos últimos 5 anos, como a recente flexibilização da carga horária semanal do médico de família e comunidade. 0 Ministério da Saúde também criou uma série de incentivos para o trabalho em municípios com dificuldade de atração ou retenção de médicos, mas ainda não interferiu na precariedade dos vínculos de trabalho. 0 Programa de Melhoria do Acesso e da Qualidade da Atenção Básica (PMAQ-AB) pode melhorar a discussão sobre o modelo de atenção primária à saúde no País, mas tem problemas técnicos e uma parte importante de seus instrumentos ainda são desconhecidos. A efetiva promoção do acesso e da qualidade passa pela imposição do limite teórico de 4 mil pessoas por equipe, e pela seleção e/ou premiação de especialistas em medicina de família e comunidade.

\section{Abstract}

The new National Primary Care Policy keeps the essence of the previous one while incorporating innovations from the past 5 years such as the recent flexibility of the weekly working hours of medical doctors. The Brazilian Ministry of Health has also created a series of working incentives for the municipalities with difficulties in attracting and/or keeping physicians, but it has not tackled the issue of labor relations precariousness yet. The National Program for Improvement in Access and Quality of Primary Care (PMAQ-AB) can contribute to the discussion on the primary health care model in Brazil; but it has technical problems, and an important part of its instruments is still unknown. The effective promotion of access and quality depends on enforcing the theoretical limit of 4,000 people per team and selecting and/or gratifying primary health care workers for their specialty in family and community medicine.

\section{Resumen}

La nueva Política Nacional de Atención Primaria (PNAP) mantiene la esencia del anterior, al mismo tiempo que incorpora las innovaciones de los últimos 5 años, como la flexibilización reciente de la carga horaria semanal del médico de la familia y comunidad. El Ministerio de Salud de Brasil también creó una serie de incentivos para el trabajo en los municipios con dificultades para atraer o retener a los médicos, pero aún no abordó la cuestión de la precariedad de las relaciones laborales. El Programa de Mejoramiento del Acceso y de la Calidad de la Atención Primaria (PMAC-AP) puede mejorar la discusión sobre el modelo de atención primaria de salud en Brasil, pero hay problemas técnicos y algunos de sus instrumentos aún son desconocidos. La promoción efectiva del acceso y de la calidad pasa por la exigencia del límite teórico de 4,000 personas por equipo, y por la selección y/o gratificación de trabajadores de atención primaria de salud por su especialidad en medicina familiar y comunitaria.

\footnotetext{
${ }^{1}$ Escola Superior de Ciências da Santa Casa de Misericórdia de Vitória (EMESCAM). leonardof@leonardof.med.br

${ }^{*}$ Autor correspondente.

Fonte de financiamento: nenhuma.

Conflito de interesses: declara não haver.

Recebido em: 28/10/2011

Aprovado em: 24/01/2012
} 


\section{Introdução}

No início de 2011, ao tomar posse, o excelentíssimo ministro da saúde Dr. Alexandre Padilha assumiu a garantia ao acesso como uma de suas prioridades e gerou polêmica ao afirmar que "Não existe modelo de atenção primária à saúde único num Brasil que é táo diverso como o nosso." ${ }^{2}$. Um ano depois, já foi publicada uma série de portarias que afetam de forma significativa a atenção primária (APS) do País, culminando em uma nova Política Nacional de Atenção Básica (PNAB).

\section{Flexibilização da carga horária semanal dos médicos de família e comunidade}

O Ministério da Saúde (MS) flexibilizou a carga horária dos médicos nas equipes de Saúde da Família, como uma forma de responder a "dificuldades de provimento e fixação" desses profissionais ${ }^{2}$. Com essa alteração da PNAB, cada equipe poderá ter um ou mais médicos, cada um deles com carga horária semanal de 20, 30 ou 40 horas. O número máximo de pessoas sob os cuidados de cada equipe (e o repasse federal) varia de acordo com a carga horária, mas cada pessoa continua devendo estar vinculada a um único profissional.

A flexibilização pode complicar em muito o processo de trabalho das unidades de Saúde da Família. Primeiro, porque médicos com até 30 horas em uma equipe poderão estar inseridos em mais de uma equipe, o que pode diminuir o vínculo das pessoas a ele vinculadas com o resto das equipes. Segundo, porque a flexibilização é restrita ao médico, dando margem a conflitos com os outros profissionais.

A flexibilização da carga horária tem sido muito criticada por vários médicos de família e comunidade (MFC) e chegou a ganhar o apelido de "portaria Samuel Blaustein", em referência ao personagem do comediante Marcos Plonka conhecido pelo bordão "Fazemos qualquer negócio!". Ao mesmo tempo, outros MFC já defendiam a flexibilizaçáo da jornada de trabalho, como discutido no número anterior da Revista Brasileira de Medicina de Família e Comunidade ${ }^{3,4}$.

Pessoalmente, acredito que o mais importante seja garantir condiçôes adequadas de trabalho, inclusive vínculo estável, para que os médicos tenham a possibilidade de se dedicar exclusivamente à Saúde da Família. Mesmo assim, a flexibilização da carga horária pode ser importante para os MFC que desejem compatibilizar a assistência à saúde com atividades de outra natureza, como a docência.

A portaria criou ainda a figura da equipe transitória, com um único médico cumprindo 20 horas e os demais profissionais, 40. Apesar de uma série de restriçóes a esse tipo de equipe, não é estipulado qualquer prazo para a resolução dessa situação "transitória". Idealmente, esse tipo de equipe nem deveria existir, mas é até razoável que exista se considerarmos a alternativa: de acordo com o diretor de atenção básica ${ }^{5}$ a atual gestão, ao assumir o mandato, teria encontrado uma portaria, pronta para ser assinada, autorizando equipes de Saúde da Família sem médico algum, por pressão dos secretários municipais de saúde do Nordeste.

\section{A nova Política Nacional de Atenção Básica}

Em menos de 2 meses de vigência, a portaria que flexibilizou a carga horária dos MFC foi revogada, ou mais apropriadamente, consolidada, pela portaria $\mathrm{n}^{\circ} 2.488$, de 21 de outubro de 2011, que instituiu a nova $\mathrm{PNAB}^{6}$. De forma geral, a nova PNAB manteve a essência da anterior ${ }^{7}$ e incorporou (além da flexibilidade de carga horária já mencionada) os núcleos de apoio à Saúde da Família (NASF) ${ }^{8-10}$, as equipes de Saúde da Família ribeirinhas e unidades de saúde fluviais ${ }^{11}$, e a presença de profissionais de saúde bucal das equipes de Saúde da Família nas equipes de agentes comunitários de saúde $(\mathrm{ACS})^{12}$, através da consolidação das respectivas portarias, além de regulamentar os Consultórios de Rua e, no âmbito da atenção primária à saúde, o Programa Saúde na Escola (PSE) ${ }^{29}$.

Apesar dos temores levantados pelo discurso de posse do ministro, a Saúde da Família continua sendo a estratégia prioritária para a APS no País. Aliás, é interessante notar que a portaria deixa explícito que o MS considera "atenção básica" um sinônimo de "atenção primária à saúde". Isto está em acordo com um documento interno de 2003, segundo o qual a adoção do termo "atenção básica" teria sido uma forma de construir "[...] uma identidade institucional própria, capaz de estabelecer uma ruptura com uma concepção redutora desse nível de atenção." ${ }^{3}$. A maior inovaçáo conceitual desta $\mathrm{PNAB}$, em relação à anterior, foi reconhecer que a APS se articula com os outros serviços de saúde na forma de redes de atenção à saúde, que nos últimos meses passaram das câmaras intergestores para a legislaçãoo ${ }^{14,15}$.

O papel do MFC na APS não sofreu nenhuma alteração significativa. Em analogia à portaria da flexibilização de carga horária, a nova PNAB especifica que as equipes deverão contar com "[...] médico generalista ou especialista em saúde da família ou médico de família e comunidade.”.

A nova PNAB mantém o limite de 4 mil pessoas adscritas a cada equipe de Saúde da Família padrão, com uma média recomendada de 3 mil pessoas, e acrescentou a recomendação de que o número de pessoas sob os cuidados de cada equipe 
seja menor em populaçóes de maior vulnerabilidade. $\mathrm{Na}$ prática, contudo, o autor deste artigo desconhece qualquer equipe que tenha sido descadastrada por ultrapassar o limite de 4 mil pessoas. O mais importante, então, não é saber qual é o limite de pessoas adscritas a cada equipe, e sim se o MS vai ou não fazer valer o limite.

\section{Saúde em todo lugar}

Desde 2010 estava previsto que os médicos devedores do Fies (Fundo de Financiamento ao Estudante do Ensino Superior) seriam beneficiados: 1) com uma prorrogação da carência, caso cursassem residência médica em "especialidades prioritárias"; e 2) com um abatimento de $1 \%$ da dívida ao mês, caso trabalhassem em municípios "com carência e dificuldade de retenção" de médicos ${ }^{16}$. Em 2011, o MS regulamentou esses benefícios: a medicina de família e comunidade e outras 20 especialidades e 4 áreas de atuação foram definidas como prioritárias, e foi compilada uma lista de 2219 municípios com escassez e rotatividade de médicos ${ }^{17,18,31}$.

Essas iniciativas são a proposta do MS e do Ministério da Educação (MEC) para a discussão sobre o Serviço Civil em Saúde ${ }^{34}$. Caso efetivamente atendam à necessidade de médicos e outros profissionais nessas áreas, é possível que enfim se abandone a antiga proposta de Serviço Civil Obrigatório, pela qual todo médico recém-formado (ao menos em escolas públicas) seria obrigado a trabalhar em áreas remotas antes de poder escolher livremente a residência médica ou o trabalho que lhe convier.

Os profissionais que atuarem na APS dos municípios com escassez e dificuldade de fixação de médicos também poderão ser incluídos no Programa de Valorização dos Profissionais da Atenção Básica (PROVAB) ${ }^{19}$. Através desse programa, médicos que trabalhem na APS desses municípios por ao menos um ano receberão um bônus na pontuação de qualquer prova de residência médica. Além disso, médicos, enfermeiros e dentistas receberão, ao longo do segundo ano de trabalho ${ }^{32}$, um curso de especialização em Saúde da Família ${ }^{33}$.

A Sociedade Brasileira de Pediatria se opôs publicamente a esse programa, destacando que o médico recém-formado não tem "a experiência e habilidade" necessárias para a APS, e que o programa não fixa o profissional na região, apesar de reconhecer a importância de se proverem médicos para o interior do País ${ }^{20}$. A Sociedade Brasileira de Medicina de Família e Comunidade destacou essencialmente os mesmos pontos, mas considerou o programa mais positivo do que negativo, e emitiu um parecer favorável com ressalvas ${ }^{21}$. É interessante observar como o programa mobilizou um consenso sobre a importância da especialização do profissional que atua na APS!

\section{Programa Nacional de Melhoria do Acesso e da Qualidade da Atenção Básica}

O Programa Nacional de Melhoria do Acesso e da Qualidade da Atenção Básica (PMAQ-AB) tem como diretriz o estímulo à mudança incremental do processo de trabalho na APS. Na fase de "desenvolvimento", as equipes realizarão uma autoavaliação (instrumento ainda não divulgado), monitorarão um determinado conjunto de indicadores de saúde e deveráo passar por educaçáo permanente e receber apoio institucional. $\mathrm{Na}$ fase de avaliação externa, o critério de certificação ficou dividido em: (10\%) verificar se as equipes implementaram a autoavaliação (independentemente do resultado); (20\%) verificar um determinado conjunto de indicadores de desempenho ("produção"); e (70\%) da avaliação consistirá em verificar "evidências para um conjunto de padrôes de qualidade" (instrumento ainda não divulgado). Os indicadores de desempenho seráo avaliados pelo próprio MS, mas os demais serão avaliados por instituiçôes de ensino e/ou pesquisa contratados pelo MS. A nota total de cada equipe será comparada com a de todas as outras equipes de municípios do mesmo grupo, e o componente de qualidade do PAB variável será definido por essa comparação. A avaliação externa também envolverá itens que não farão parte da certificação (e, portanto, não contarão para o incentivo), mas que deverão subsidiar a gestão, como a satisfação do usuário ${ }^{22-24}$.

Do ponto de vista técnico, uma vez que já existem instrumentos consagrados e validados como o $\mathrm{AMQ}^{25}$ e o Primary Care Assessment Tool (PCATool) ${ }^{26}$, fica difícil entender a necessidade de se criar um novo instrumento ${ }^{30}$, ainda que baseado em instrumentos pré-existentes. Os próprios indicadores de saúde do PMAQ-AB são muitas vezes questionáveis, como, por exemplo, o coeficiente de preventivos de câncer de útero realizados em mulheres de 15 anos ou mais - o Inca (Instituto Nacional de Câncer) recomenda o rastreamento apenas em mulheres de 25 aos 64 anos de idade ${ }^{27}$; e colher um preventivo de seis em seis meses numa mulher nunca deveria ser equiparado a colher um preventivo de 3 em 3 anos em 6 mulheres. Mas a maior questão é: que conjunto de instituições de ensino será capaz de verificar a qualidade de todas as dezenas de milhares de equipes do País?

Como o PMAQ-AB não será restrito às equipes de Saúde da Família, o MS criou a figura das "equipes de atenção básica" $(\mathrm{EAB})^{28}$. Para ser inscrita no PMAQ-AB, uma EAB precisará contar com pelo menos um médico generalista ou clínico (não MFC), um pediatra, um enfermeiro, um auxiliar ou técnico de enfermagem, e um ACS; o ginecologista é opcional. Dependendo da carga horária de cada profissional, a equipe pode ser equiparada a uma, duas ou três equipes 
padrão de Saúde da Família; se houver cirurgião-dentista, pode ser equiparada à equipe de saúde bucal.

São necessárias, por exemplo, 100 horas médicas numa EAB para equipará-la a uma equipe de Saúda da Família, com 40 horas. Dessa forma, a criação das EAB permite que o MS induza qualidade mesmo na APS não Saúde da Família, ao mesmo tempo em que a Saúde da Família persiste como a estratégia preferencial. Além disso, com uma participação mais expressiva do enfermeiro e a obrigatoriedade do ACS, a conformação em EAB deverá induzir a APS a ficar, cada vez mais, semelhante ao que preconiza a estratégia Saúde da Família.

\section{Conclusão}

Apesar das preocupaçóes decorrentes da afirmação do ministro em seu discurso de posse, a Saúde da Família continua sendo a estratégia prioritária para a organização da APS no Brasil. Em termos práticos, o MFC continua tendo o mesmo papel, mas agora é explicitamente mencionado na redação da $\mathrm{PNAB}$.

Apesar das limitaçóes e, principalmente, das incertezas acerca do PMAQ-AB, a avaliação sistemática da APS tem o potencial de reafirmar a superioridade da estratégia Saúde da Família, ao mesmo tempo que nos permite verificar o que pode e o que não pode ser negociado. Mesmo assim, náo deixa de ser desconcertante que o MS não tenha simplesmente adotado um instrumento de avaliação abrangente e validado como o PCATool.

No que diz respeito à garantia de acesso, o PROVAB e os incentivos aos devedores do Fies certamente beneficiarão os municípios com falta de médicos, mas a efetividade de se flexibilizar a carga horária dos médicos em equipes de Saúde da Família é algo que só o tempo poderá mostrar.

Mas não basta que o município conte com médicos; é preciso que o número de pessoas sob os cuidados de cada médico também seja adequado. Enquanto muitos MFC defendem o limite de 2 mil pessoas por médico, o MS já estaria fazendo um grande progresso se começasse a exigir o cumprimento do limite de 4 mil.

O governo federal esboça uma série de iniciativas relativas ao provimento de recursos humanos, como o aumento das vagas de graduação e residência médicas, mas, para efetivamente fortalecer a APS, são necessárias iniciativas do outro lado, como a valorização dos especialistas em APS, a desprecarização dos vínculos de trabalho, e a instituição de um plano de carreira adequado.

\section{Referências}

1. Brasil. Ministério da Saúde. Íntegra do discurso do ministro da saúde, Alexandre Padilha, durante a cerimônia de transmissão de cargo, em 3 de janeiro de 2011, em Brasília (DF) [online]. Brasília: Ministério da Saúde; 2011. Disponível em: http://portal.saude.gov. br/portal/aplicacoes/noticias/default.cfm?pg=dspDetalheNoticia\&id_ area $=1450 \&$ CO_NOTICIA $=12054$

2. Brasil. Ministério da Saúde. Gabinete do Ministro. Portaria n 2.027, de 25 de agosto de 2011. Altera a Portaria n 648/GM/MS, de 28 de março de 2006, na parte que dispõe sobre a carga horária dos profissionais médicos que compõem as Equipes de Saúde da Família - ESF e na parte que dispõe sobre a suspensão do Piso de Atenção Básica - PAB variável. Diário Oficial da República Federativa do Brasil, Brasília, DF, 25 ago. 2011.

3. Silva FD. 40 horas na ESF? Rev Bras Med Fam Comunidade. 2011; 6(18): 15-6.

4. Lopes JMC. 40 horas na ESF? Rev Bras Med Fam Comunidade. 2011; 6(18): 17-8.

5. Brasil. Ministério da Saúde. Comentários à portaria sobre a carga horária de médicos no PSF. In: Freitas GC. Primeiros Cuidados com Saúde: Atenção Básica e a Atenção Primária [Internet]. Blog Saúde com Dilma; 2010. [citado em 2011 out 24]. Disponível em: http://www. saudecomdilma.com.br/index.php/2011/08/28/primeiros-cuidadoscom-saude-atencao-basica-e-a-atencao-primaria/

6. Brasil. Ministério da Saúde. Gabinete do Ministro. Portaria n².488, de 21 de outubro de 2011. Aprova a Política Nacional de Atenção Básica, estabelecendo a revisão de diretrizes e normas para a organização da Atenção Básica, para a Estratégia Saúde da Família - ESF e o Programa de Agentes Comunitários de Saúde - PACS. Diário Oficial da República Federativa do Brasil, Brasília, DF, 24 out. 2011.

7. Brasil. Ministério da Saúde. Gabinete do Ministro. Portaria n 648, de 28 de março de 2006. Aprova a Política Nacional de Atenção Básica, estabelecendo a revisão de diretrizes e normas para a organização da Atenção Básica para o Programa Saúde da Família (PSF) e o Programa Agentes Comunitários de Saúde (PACS). Diário Oficial da República Federativa do Brasil, Brasília, DF, 29 mar. 2006.

8. Brasil. Ministério da Saúde. Gabinete do Ministro. Portaria n 154 de 24 de janeiro de 2008. Cria os Núcleos de Apoio à Saúde da Família - NASF. Diário Oficial da República Federativa do Brasil, Brasília, DF, 2008 jan 25; 145(18):47.

9. Brasil. Ministério da Saúde. Gabinete do Ministro. Portaria n 2.281, de $1^{\circ}$ de outubro de 2009. Altera a Portaria n 154/GM, de 4 de março de 2008, que cria os Núcleos de Apoio à Saúde da Família - NASF. Diário Oficial da República Federativa do Brasil, Brasília, DF, 02 out. 2010.

10. Brasil. Ministério da Saúde. Gabinete do Ministro. Portaria $n^{\circ} 2.843$, de 20 de setembro de 2010. Cria, no âmbito do Sistema Único de Saúde - SUS, os Núcleos de Apoio à Saúde da Família - Modalidade 3 - NASF 3, com prioridade para a atenção integral para usuários de crack, álcool e outras drogas. Diário Oficial da República Federativa do Brasil, Brasília, DF, 21 set. 2010.

11. Brasil. Ministério da Saúde. Gabinete do Ministro. Portaria n 2.191, de 3 de agosto de 2010. Institui critérios diferenciados com vistas à implantação, financiamento e manutenção da Estratégia de Saúde da Família para as populações ribeirinhas na Amazônia Legal e em Mato Grosso do Sul. Diário Oficial da República Federativa do Brasil, Brasília, DF, 04 ago. 2009.

12. Brasil. Ministério da Saúde. Gabinete do Ministro; Portaria $n^{\circ} 302$, de 3 de fevereiro de 2009. Estabelece que profissionais de Saúde Bucal da Estratégia Saúde da Família poderão ser incorporados às Equipes 
de Agentes Comunitários de Saúde EACS. Diário Oficial da República Federativa do Brasil, Brasília, DF, 18 fev. 2009.

13. Brasil. Ministério da Saúde. Documento Final da Comissão de Avaliação da Atenção Básica [online]. Brasília: Ministério da Saúde; 2003. Disponível em: http://www.saude.sc.gov.br/gestores/sala_de_leitura/ artigos/atencao_basica_bibliografias/Documento_Final_da_Comissao_ de_Avaliacao_Atencao_Basica.pdf

14. Brasil. Presidência da República. Decreto $n^{\circ} 7.508$, de 28 de junho de 2011. Regulamenta a Lei $n^{\circ} 8.080$, de 19 de setembro de 1990, para dispor sobre a organização do Sistema Único de Saúde - SUS, o planejamento da saúde, a assistência à saúde e a articulação interfederativa, e dá outras providências. Diário Oficial da República Federativa do Brasil, Brasília, DF, 29 jun. 2011.

15. Brasil. Ministério da Saúde. Gabinete do Ministro. Portaria n 4.279, de 30 de dezembro de 2010. Estabelece diretrizes para a organização da Rede de Atenção à Saúde no âmbito do Sistema Único de Saúde - SUS. Diário Oficial da República Federativa do Brasil, Brasília, DF, 31 dez. 2010.

16. Brasil. Lei $n^{\circ} 12.202$, de 14 de janeiro de 2010. Altera a Lei $n^{\circ} 10.260$, de 12 de julho de 2001, que dispõe sobre o Fundo de Financiamento ao Estudante do Ensino Superior - FIES (permite abatimento de saldo devedor do FIES aos profissionais do magistério público e médicos dos programas de saúde da família; utilização de débitos com o INSS como crédito do FIES pelas instituições de ensino; e dá outras providências). Diário Oficial da República Federativa do Brasil, Brasília, DF, 15 jan. 2010.

17. Brasil. Ministério da Saúde. Gabinete do Ministro. Portaria no 1.377 , de 13 de junho de 2011. Estabelece critérios para definição das áreas e regiões prioritárias com carência e dificuldade de retenção de médico integrante de equipe de saúde da família oficialmente cadastrada e das especialidades médicas prioritárias de que tratam o inciso II e o $\S 3^{\circ}$ do art. $6^{\circ}$-B da Lei no 10.260, de 12 de julho de 2001, no âmbito do Fundo de Financiamento ao Estudante do Ensino Superior (FIES) e dá outras providências. Diário Oficial da República Federativa do Brasil, Brasilia, DF, 14 jun. 2011.

18. Brasil. Ministério da Saúde. Secretaria de Atenção à Saúde. Portaria Conjunta $n^{\circ} 2$, de 25 de agosto de 2011. Define os Municípios priorizados e a relação das especialidades médicas e áreas de atuação segundo os critérios dispostos na Portaria $n^{\circ} 1.377 / 2011$, para fins do benefício previsto no inciso II e o $\S 3^{\circ}$ do art. $6^{\circ} \mathrm{B}$ da Lei $\mathrm{n}^{\circ} 10.260$, de 12 de julho de 2001, no âmbito do Fundo de Financiamento ao Estudante do Ensino Superior (FIES). Diário Oficial da República Federativa do Brasil, Brasília, DF, 26 ago. 2011.

19. Brasil. Ministério da Saúde. Gabinete do Ministro. Portaria Interministerial $n^{\circ} 2.087$, de 01 de setembro de 2011. Institui o Programa de Valorização do Profissional da Atenção Básica. Diário Oficial da República Federativa do Brasil, Brasília, DF, 02 set. 2011.

20. Sociedade Brasileira de Pediatria - SBP. Conselho Superior e Fórum de Defesa Profissional. Posicionamento da Sociedade Brasileira de Pediatria frente ao Programa de Valorização do Profissional da Atenção Básica e à formação do pediatra [online]. Salvador: SBP; 2011. Disponível em: http://www.sbp.com.br/show_item2. cfm?id_categoria $=52 \& i d \_d e t a l h e=4002 \&$ tipo_detalhe $=$ s

21. Sociedade Brasileira de Medicina de Família e Comunidade - SBMFC. SBMFC emite parecer oficial quanto ao PL 1363/11 [online]. Florianópolis: SBMFC; 2011. Disponível em: http://www.sbmfc.org. $\mathrm{br} /$ default.asp?site_Acao=MostraPagina\&Paginald $=11 \& \mathrm{mNoti} \_$Acao $=$ mostraNoticia\&noticiald $=414$

22. Brasil. Ministério da Saúde. Gabinete do Ministro. Portaria n 1.654 , de 19 de julho de 2011. Institui, no âmbito do Sistema Único de Saúde, o Programa Nacional de Melhoria do Acesso e da Qualidade da Atenção Básica (PMAQ-AB) e o Incentivo Financeiro do PMAQ-AB, denominado Componente de Qualidade do Piso de Atenção Básica Variável - PAB Variável. Diário Oficial da República Federativa do Brasil, Brasília, DF, 20 jul. 2011.

23. Brasil. Ministério da Saúde. Gabinete do Ministro. Portaria n².396, de 13 de outubro de 2011. Define o valor mensal integral do incentivo financeiro do (PMAQ-AB), denominado componente de qualidade do piso de atenção básica variável PAB variável. Diário Oficial da República Federativa do Brasil, Brasília, DF, 14 out. 2011.

24. Brasil. Ministério da Saúde. Secretaria de Atenção à Saúde. Departamento de Atenção Básica. Programa Nacional de Melhoria do Acesso e da Qualidade da Atenção Básica (PMAQ): Manual Instrutivo [online]. Brasília: Ministério da Saúde; 2011. Disponível em: http://189.28.128.100/dab/docs/sistemas/Pmaq/pmaq_manual_ instrutivo.pdf

25. Brasil. Ministério da Saúde. Secretaria de Atenção à Saúde. Departamento de Atenção Básica. Avaliação para melhoria da qualidade da estratégia saúde da família. Brasília: Ministério da Saúde; 2005.

26. Brasil. Ministério da Saúde. Secretaria de Atenção em Saúde. Departamento de Atenção Básica. Manual do instrumento de avaliação da atenção primária à saúde: primary care assessment tool pcatool - Brasil. Brasília: Ministério da Saúde; 2010.

27. Instituto Nacional de Câncer - INCA. Coordenação Geral de Ações Estratégicas. Divisão de Apoio à Rede de Atenção Oncológica. Diretrizes brasileiras para o rastreamento do câncer do colo do útero. Rio de Janeiro: INCA; 2011.

28. Brasil. Ministério da Saúde. Secretaria de Atenção em Saúde. Portaria $n^{\circ}$ 576, de 19 de setembro de 2011. Estabelece novas regras para a carga horária semanal (CHS) dos profissionais médicos, enfermeiros e cirurgião-dentista, conforme descrito no Anexo I. Diário Oficial da República Federativa do Brasil, Brasília, DF, 19 set. 2011.

29. Brasil. Presidência da República. Decreto $n^{\circ} 6.286$, de 5 de dezembro de 2007. Institui o Programa Saúde na Escola - PSE, e dá outras providências. Diário Oficial da República Federativa do Brasil, Brasília, DF, 06 dez. 2007.

30. Brasil. Ministério da Saúde. Secretaria de Atenção à Saúde. Departamento de Atenção Básica. Auto-avaliação para a melhoria do acesso e da qualidade da atenção básica - AMAQ [online] Brasília: Ministério da Saúde; 2012. No prelo. Disponível em: http://189.28.128.100/dab/docs/sistemas/Pmaq/amaq_2011.pdf

31. Brasil. Ministério da Saúde. Secretaria de Atenção à Saúde. Portaria Conjunta $n^{\circ} 3$, de 01 de novembro de 2011. Acresce inciso III ao art. $3^{\circ}$ e altera o parágrafo único do art. $4^{\circ}$ da Portaria Conjunta $n^{\circ} 2 /$ SAS/SGTES, de 25 de agosto de 2011. Diário Oficial da República Federativa do Brasil, Brasília, DF, 03 nov. 2011.

32. Brasil. Ministério da Saúde. Gabinete do Ministro. Edital $n^{\circ} 8$, de 07 de dezembro de 2011. Programa de Valorização do Profissional da Atenção Básica - PROVAB. Diário Oficial da República Federativa do Brasil, Brasília, DF, 08 dez. 2011.

33. Brasil. Ministério da Saúde. Gabinete do Ministro. Edital n 1, de 09 de janeiro de 2012. Processo seletivo para escolha de egressos de instituições de ensino superior. Diário Oficial da República Federativa do Brasil, Brasília, DF, 10 jan. 2012.

34. Brasil. Conselho Nacional de Saúde. Seminário Nacional sobre Serviço Civil em Saúde e Demandas Judiciais no Âmbito do SUS [online]. Brasília: Conselho Nacional de Saúde; 2011. Relatório Final. Disponível em: http://conselho.saude.gov.br/biblioteca/Relatorios/ relatorio_seminario_judicializacao.pdf 